American Journal of Applied Sciences 8 (1): 63-70, 2011

ISSN 1546-9239

(C) 2010 Science Publications

\title{
Comparison and Analysis of Discrete Cosine Transform based Joint Photographic Experts Group Image Compression using Robust Watermarking Algorithm
}

\author{
S.M. Ramesh and Dr. A. Shanmugam \\ Department of Electronics and Communication Engineering, \\ Bannari Amman Institute of Technology, \\ Sathyamangalam-Erode- 638401, Tamilnadu, India
}

\begin{abstract}
Problem statement: The performance of the watermarking algorithms is analyzed using different compression standards to study the watermark extraction behavior of these algorithms. Approach: A digital watermarking method is said to be effective if the watermark embedded in an image by it could survive against diverse attacks ranging from compression, filtering to cropping. Several techniques are presented in the literature for robust watermarking algorithm to defend against the various the compression methods. Results: In this study, we have presented comparison and analysis of recently developed watermarking algorithms. Then, an extensive analysis is carried out to estimate and compare robustness of watermarking algorithms by considering the visual quality of the original and watermarked images in terms of Peak Signal to Noise Ratio. Furthermore, the extracting fidelity of the watermarking algorithms is compared by taking the Normalized Correlation value between the original watermark and the extracted watermark. Conclusion/Recommendations: The experimental results showed the accuracy of different watermarking algorithms in terms of visual quality and fidelity.
\end{abstract}

Key words: Digital watermarking, robust watermarking, image Compression, Discrete Cosine Transform (DCT), Joint Photographic Experts Group (JEPG), Peak Signal to Noise Ratio (PSNR), Normalized Correlation (NC), diverse applications, Human Visual System (HVS), digital watermarking, spatial-domain, Kernel Fuzzy C-Means (KFCM)

\section{INDRODUCTION}

Digital watermarking is a technique used to protect digital images from illegal copying and exploitation. Data is embedded into a multimedia element like image, audio or video by the process of watermarking. Diverse applications such as copyright protection, access control and broadcast monitoring use this embedded data extracted or detected from the multimedia element (Cox et al., 2002). Digital watermarking has attracted the attention of a considerable number of researchers in the period between early to mid-1990s. From that time onwards the number of publications per year has increased rapidly to several hundred (Petitcolas et al., 1999). It began from simple approaches offering the basic principles to highly-developed communication theory results oriented algorithms and applying them for the watermarking problem (Surekha et al., 2009; Bankovic et al., 2009; Szczypiorski et al., 2007;
Piotrowski and Gajewski, 2007; Sarhan, 2009; Marimuthu and Thangaraj, 2010). Cover image, watermark structure, embedding algorithm and extraction or detection algorithm are the basic components of an image watermarking algorithm (Lai et al., 2009).

Watermarking requirements: The following requirements must be fulfilled by a good watermarking system (Langelaar et al., 2000):

- Transparency: The degree of degradation of the quality of the quality due to the embedded watermark must be minimal

- Robustness: Common image processing operations such as cropping, rotation, filtering and compression should not affect the embedded watermark

- Security: Even if the embedding algorithm is made public, the watermarking scheme must be secure. Cryptographic techniques are commonly employed to accomplish security

Corresponding Author: S.M. Ramesh, Department of Electronics and Communication Engineering,

Bannari Amman Institute of Technology, Sathyamangalam-Erode- 638401, Tamilnadu, India 
Am. J. Applied Sci., 8 (1): 63-70, 2011

- Appropriate complexity: The compression/decompression processes should require small amount of computation and memory, particularly for real time application

Careful design is required for tradeoff because it is evident that these requirements may conflict with each other. In general, Watermarks and watermarking methods can be classified into different categories in several ways. Digital watermarking can be classified into image watermarking, video watermarking and audio watermarking (Wang and Zhao, 2006) on the basis of the range of application. Watermarking techniques are broadly classified into spatial-domain and frequency-domain methods (Kumar et al., 2009) depending on the domain in which the watermark is inserted.

Previous study: A handful of watermarking schemes have been presented in the literature for protecting the copyrights of digital images. Recently, robust watermarking schemes have received a great deal of attention among watermarking researchers because of its performance and effectiveness. A brief review of some recent researches is presented here.

Bohra et al. (2009) have proposed a technique based on second-generation wavelets (lifting-based integer wavelets transform) for robust watermarking of images. The capability of blind self-authentication of the watermarked image has been present in the proposed approach along with robustness. Due to the employment of integer-to-integer transform, the watermarked images have not shown any perpetual degradation and a Peak Signal To Noise Ratio (PSNR) in excess of $40 \mathrm{~dB}$ has been obtained. For diverse attacks for example filtering, compression and rotation, the proposed technique has shown superior performance over other similar existing schemes, in the simulation results. Also, alterations in the image generates dissimilar sequences because of the two independent processes that has been employed in the proposed watermark sequence extraction, thereby alterations in the image have been detected.

MeenakshiDevi et al. (2009) have introduced an adaptive image watermarking approach which comprises Kernel Fuzzy C-Means (KFCM) clustering algorithm and Human Visual System (HVS). First they have performed block-wise DCT transform after segmenting the host image into image blocks. Then, in order to choose the embedding position and find out the embedding strength of image blocks adaptively they have trained the KFCM using the three local features of image blocks extracted from its image coefficients. The robustness of the proposed algorithm to common attacks for example JPEG, filtering, noise addition, scaling, sharpen, has been confirmed by experimental results.

Lu et al. (2010) have presented a robust, feature point detection and image normalization based watermarking scheme. First, using the proposed multiresolution feature point detection filter, they have identified some stable feature points from the original image. Then, image normalization has been performed on the disks centered at these feature points. For each disk the watermark has been embedded separately in the sub band coefficients of DFT domain. The original image has not been required and the correlation between the watermark embedding coefficients and the original watermark has been used by the watermark detection process. The proposed scheme which achieved strong robustness to signal processing and geometrical distortions has combined the advantages of feature point detection and image normalization. The superior performance of the proposed scheme has been demonstrated by experimental results.

Watermarking algorithms: The main objective of the proposed research is to compare the watermarking algorithms described in the literature (Liu et al., 2006; Ye and Tan, 2008; Kung et al., 2009; Lin et al., 2010; Chandra et al., 2010; Coria et al., 2007). Algorithms proposed to perform robust watermarking in the frequency domain are selected for comparison. Their robustness is obtained for several compression standards and the results are extensively analyzed for diverse compression ratios. The following subsections provide a detailed description of the embedding and extracting process of the watermarking algorithms.

Liu et al. (2006) algorithm: Liu et al. (2006) have proposed a self-reference image based robust watermarking scheme. In order to overcome the weak robustness problem in spatial domain, their robust watermarking scheme has modified the original image in transform domain and has embedded the watermark in the difference values between the original image and its reference image. Furthermore, the application has been more practical in real life application for ownership verification because original images have not been required for watermark extraction.

Ye and Tan (2008) algorithm: Ye and Tan (2008) have proposed an improved digital watermarking algorithm for a meaningful image. They have processed the image using Arnold transformation and correlation detection and multiplied the spectrum of the watermarking with the pseudo-random series to obtain the private key. 
Am. J. Applied Sci., 8 (1): 63-70, 2011

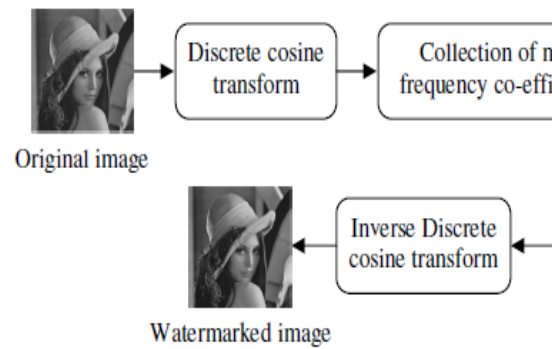

Watermarked image

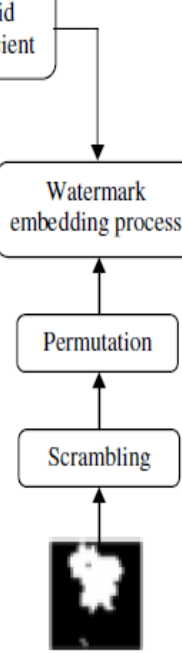

Watermark image

Fig. 1: Watermark embedding process

Kung et al. (2009) algorithm: Kung et al. (2009) have proposed a robust frequency domain watermarking scheme. The coefficients of the middle frequency have been employed by their algorithm for oblivious watermarking.

\section{Watermark embedding steps:}

Step 1: Obtain $\mathrm{X}_{\mathrm{f}}$ by performing block transformation of the host image $f$ by means of DCT. To be consistent with the JPEG compression standard, they choose $8 \times 8$ as the block size and serially label the transformed blocks as $B_{k}$ for $0 \leq \mathrm{j}<\mathrm{N} \times \mathrm{N} / 64$.

Step 2: Find 16 coefficients out of the middle frequency coefficients for each $8 \times 8$ block $B_{i}$. Append eight of them to the sequence $a_{1}(j)$ and the remaining eight to $a_{2}(j)$ and select the sequence $\mathrm{q}_{1}$ and $\mathrm{q}_{2}$ corresponding to $\mathrm{a}_{1}$ and $\mathrm{a}_{2}$ from the quantization table.

Step 3: Obtain $\mathrm{w}_{\mathrm{s}}$ by performing the scrambling operation on the watermark $\mathrm{w}_{\mathrm{g}}$ using the relation, $\mathrm{w}_{\mathrm{s}}=\mathrm{w}_{\mathrm{g}} \oplus \mathrm{c}$, where $\mathrm{c}$ is the chaotic binary sequence of size $\mathrm{M} \times \mathrm{M}$.

Step 4: Perform the permuting operation on the scrambled watermark $\mathrm{w}_{\mathrm{s}}$ to obtain $\mathrm{w}_{\mathrm{p}}$.

Step 5: Obtain $\mathrm{w}_{\mathrm{p}}$ by carrying out the permuting operation on the scrambled watermark.

Step 6: Using the bipolar function, transform the watermark value. The watermark value is defined in the bipolar form $\{-1,1\}$ as:

$\mathrm{w}_{\mathrm{b}}(\mathrm{x}, \mathrm{y})=\left\{\begin{array}{rc}1, & \text { if } \mathrm{w}_{\mathrm{p}}(\mathrm{x}, \mathrm{y})=1 \\ -1, & \text { else }\end{array}\right.$

Step 7: Modify the image $\mathrm{X}_{\mathrm{f}}$ based on $\mathrm{w}_{\mathrm{b}}$ as follows If watermark bit $=1(\mathrm{a}=1)$, the position corresponding to the original image is not altered.

If watermark bit $=0(\mathrm{a}=-1)$ :

$\mathrm{p}=\left|\mathrm{s}_{1}-\mathrm{s}_{2}\right| ; \mathrm{s}_{1}=\mathrm{a}_{1}(\mathrm{j}) / \mathrm{q}_{1}(\mathrm{j}), \mathrm{s}_{2}=\mathrm{a}_{2}(\mathrm{j}) / \mathrm{q}_{2}(\mathrm{j})$;

$\mathrm{t}_{1}=\mathrm{s}_{1}+\alpha \times\left\lfloor\frac{\mathrm{p}}{2}\right\rfloor+\alpha \times(\mathrm{p} \bmod 2)$

$\mathrm{t}_{2}=\mathrm{s}_{2}-\alpha \times\left\lfloor\frac{\mathrm{p}}{2}\right\rfloor-\alpha \times\left(1-\mathrm{w}_{\mathrm{s}}(\mathrm{l})\right)$;

$a_{1}{ }^{\prime}(j)=t_{1} \times q_{1}(j) ; a_{2}{ }^{\prime}(j)=t_{2} \times q_{2}(j)$

Step 8: Obtain $G$ by replacing the sequences $A_{j}(1)$ into $\mathrm{X}_{\mathrm{f}}$.

Step 9: Obtain the watermarked image g by performing the inverse block DCT on G.

The block diagram of the watermark embedding process is given in Fig. 1.

\section{Watermark extraction steps:}

Step 1: Obtain $G$ and collect the mid frequency region of $G$ as sequences $a_{1}^{\prime}(j)$ and $a_{2}^{\prime}(j)$ by performing the block transform of the watermarked image g using DCT.

Step 2: Using the demodulation function, retrieve the permuted watermark as:

$\mathrm{w}_{\mathrm{b}, \mathrm{j}}{ }^{\prime}(\mathrm{l})=\alpha=\operatorname{sign}\left(\left|\mathrm{a}_{1}^{\prime}(\mathrm{j})\right|-\left|\mathrm{a}_{2}^{\prime}(\mathrm{j})\right|\right)$

Step 3: Using the inverse bipolar function, inverse the watermark value as:

$\mathrm{w}_{\mathrm{p}}^{\prime}(\mathrm{x}, \mathrm{y})=$ inbipolar $\left[\mathrm{w}_{\mathrm{b}}^{\prime}(\mathrm{x}, \mathrm{y})\right]$

where, $\left.0 \leq \mathrm{x}, \mathrm{y}<\mathrm{M}, \mathrm{w}_{\mathrm{p}}^{\prime}(\mathrm{x}, \mathrm{y}) \in\{-1,1)\right\}$ in bipolar:

$$
(w)=\left\{\begin{array}{l}
1, w=1 \\
0, w=-1
\end{array}\right.
$$

Step 4: Apply the transform on the pixel positions of $\mathrm{w}_{\mathrm{p}}^{\prime}$ and obtain the reverse permutation.

Step 5: Using the chaotic binary sequence c, reverse the scrambling process to obtain extracted watermark w $\mathrm{g}_{\mathrm{g}}^{\prime}$ by, $\mathrm{w}_{\mathrm{g}}^{\prime}=\mathrm{w}_{\mathrm{s}}^{\prime} \oplus \mathrm{c}$. 
Am. J. Applied Sci., 8 (1): 63-70, 2011

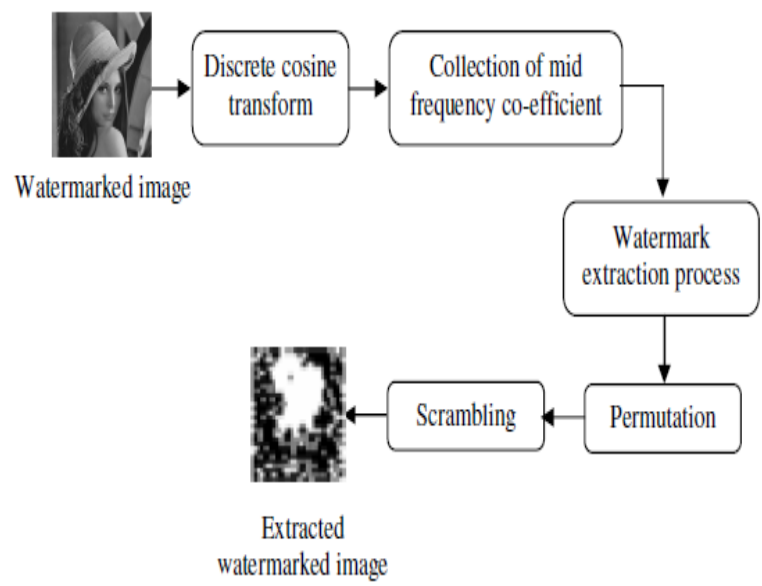

Fig. 2: Watermark extraction process

The block diagram of the watermark extraction process is given in Fig. 2.

Lin et al. (2010) algorithm: Lin et al. (2010) have proposed a watermarking technique that adjusts the DCT low-frequency coefficients by the concept of mathematical remainder to preserve acceptable visual quality of watermarked images.

\section{Watermark embedding steps:}

Step 1: Permute the watermark before it is embedded into the host image using Torus automorphism permutation as follows:

$\left(\begin{array}{l}i^{*} \\ j^{*}\end{array}\right)=\left(\begin{array}{cc}1 & i \\ k & k+1\end{array}\right) \times\left(\begin{array}{l}i \\ j\end{array}\right) \bmod m$

Step 2: Divide the luminance $\mathrm{Y}$ of original image into non-overlapping blocks of size $8 \times 8$, after YUV color transformation and DCT transform each Y block independently.

Step 3: Quantize each DCT-transformed block of Y on the basis of the quantization table provided by the JPEG compression standard, to enhance the robustness of the watermark against the JPEG compression.

Step 4: Sort the image blocks in the descending order of their respective $\mathrm{M}(\mathrm{m})$ i.e., the number of non-zero coefficients in block $n$.

Step 5: Modify the DCT coefficients at the lowfrequency positions to embed the watermark information. The variables relative to the DCT coefficient value $\mathrm{C}$ and modulus $\mathrm{N}$ can be defined as:

$$
\begin{aligned}
& p=|C| \bmod N ; q=\frac{|C|}{N} ; \\
& \text { sign }= \begin{cases}1 & \text {,if } C \geq 0 \\
-1 & \text {,if } C<0\end{cases}
\end{aligned}
$$

By changing the value of coefficient $\mathrm{C}$, the binary information of watermark pattern is embedded into the selected block. Let $\mathrm{C}^{*}$ be the value of modified coefficient corresponding to $\mathrm{C}$ can be obtained by the following rule.

If watermark bit $=0$ :

$$
\begin{aligned}
& \mathrm{p}^{\prime}=\frac{\mathrm{N}}{4} ; \mathrm{C}_{\text {low }}=\operatorname{sign} \times\left(\mathrm{q} \times \mathrm{N}+\mathrm{p}^{\prime}\right) ; \\
& \mathrm{C}_{\text {high }}=\operatorname{sign} \times\left((\mathrm{q}+1) \times \mathrm{N}+\mathrm{p}^{\prime}\right) \\
& \mathrm{C}^{*}= \begin{cases}\mathrm{C}_{\text {low }} & \text {,if }\left|\mathrm{C}_{\text {low }}-\mathrm{C}\right| \leq\left|\mathrm{C}_{\text {high }}-\mathrm{C}\right| \\
\mathrm{C}_{\text {high }} & \text {,if }\left|\mathrm{C}_{\text {low }}-\mathrm{C}\right|>\left|\mathrm{C}_{\text {high }}-\mathrm{C}\right|\end{cases}
\end{aligned}
$$

If watermark bit $=0$ :

$$
\begin{aligned}
& \mathrm{p}^{\prime}=\frac{3 \mathrm{~N}}{4} ; \mathrm{C}_{\text {low }}=\operatorname{sign} \times\left((\mathrm{q}-1) \times \mathrm{N}+\mathrm{p}^{\prime}\right) ; \\
& \mathrm{C}_{\text {high }}=\operatorname{sign} \times\left(\mathrm{q} \times \mathrm{N}+\mathrm{p}^{\prime}\right) \\
& \mathrm{C}^{*}= \begin{cases}\mathrm{C}_{\text {low }} & \text {,if }\left|\mathrm{C}_{\text {low }}-\mathrm{C}\right| \leq\left|\mathrm{C}_{\text {high }}-\mathrm{C}\right| \\
\mathrm{C}_{\text {high }} & \text {,if }\left|\mathrm{C}_{\text {low }}-\mathrm{C}\right|>\left|\mathrm{C}_{\text {high }}-\mathrm{C}\right|\end{cases}
\end{aligned}
$$

Step 6: Inverse DCT transform is applied on each block of the host image independently after all the watermark bits are inserted into the selected image blocks.

Step 7: Apply the weighted correction procedure to further improve the visual quality of the watermarked image, which makes a slight change on the watermarked (modified) luminance $\mathrm{Y}^{*}$. The weighted correction procedure can be represented in terms of the difference $\mathrm{D}$ between the original luminance $\mathrm{Y}$ and the watermarked luminance $\mathrm{Y}^{*}$ as:

$\mathrm{D}=\mathrm{Y}-\mathrm{Y}^{*} ; \mathrm{Y}^{* *}=\mathrm{Y}^{*}+\mathrm{f} \times \mathrm{D}$

Step 8: Finally, transform the YUV color planes of the watermarked image back to the original RGB color planes.

The block diagram of the watermark embedding process is given in Fig. 3. 
Am. J. Applied Sci., 8 (1): 63-70, 2011

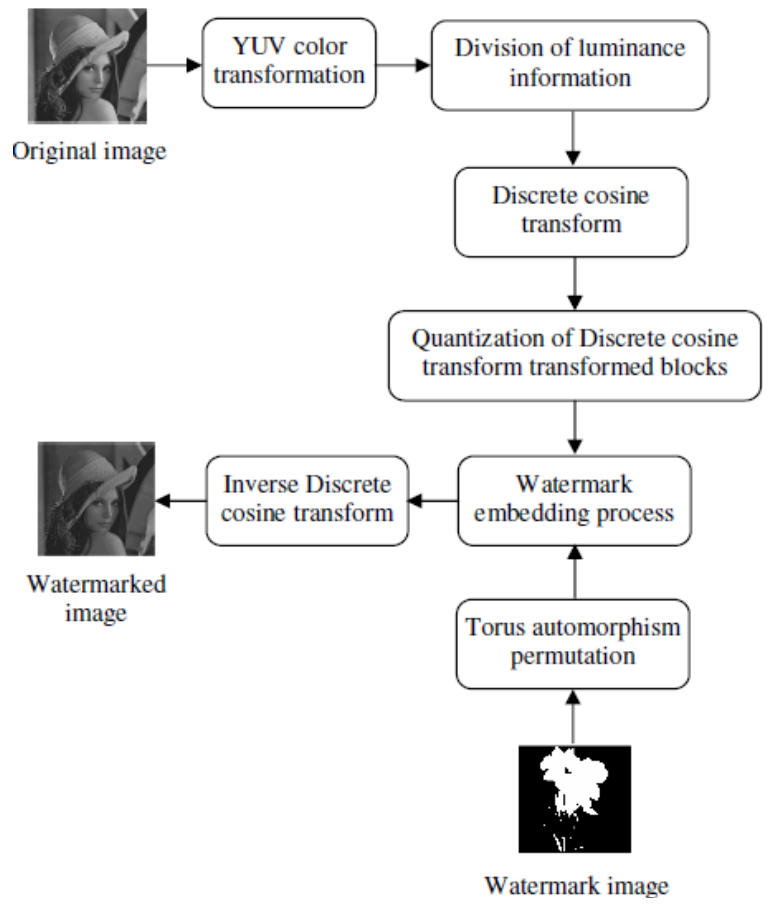

Fig. 3: Watermark embedding process

\section{Watermark extraction steps:}

Step 1: Based on the YUV color transformation, extract the luminance information of watermarked image.

Step 2: Divide the luminance information of watermarked image into non-overlapping blocks of size $8 \times 8$. Transform each block into DCT frequency domain independently based on the DCT Transformation. Generate nonoverlapping blocks of size $8 \times 8$, by dividing the luminance information of watermarked image. Based on the DCT Transformation, independently transform each block into DCT frequency domain.

Step 3: Based on the following rule, extract the binary information of the watermark:

$\mathrm{b}= \begin{cases}0 & \text {,if } \quad(|\mathrm{C}(\mathrm{i}, \mathrm{j})| \bmod \mathrm{N})<\frac{\mathrm{N}}{2} \\ 1 & \text {,if } \quad(|\mathrm{C}(\mathrm{i}, \mathrm{j})| \bmod \mathrm{N}) \geq \frac{\mathrm{N}}{2}\end{cases}$

Step 4: Using Torus auto-morphism permutation, rearrange the extracted watermark bits in the proper order and after that reconstruct the watermark pattern.

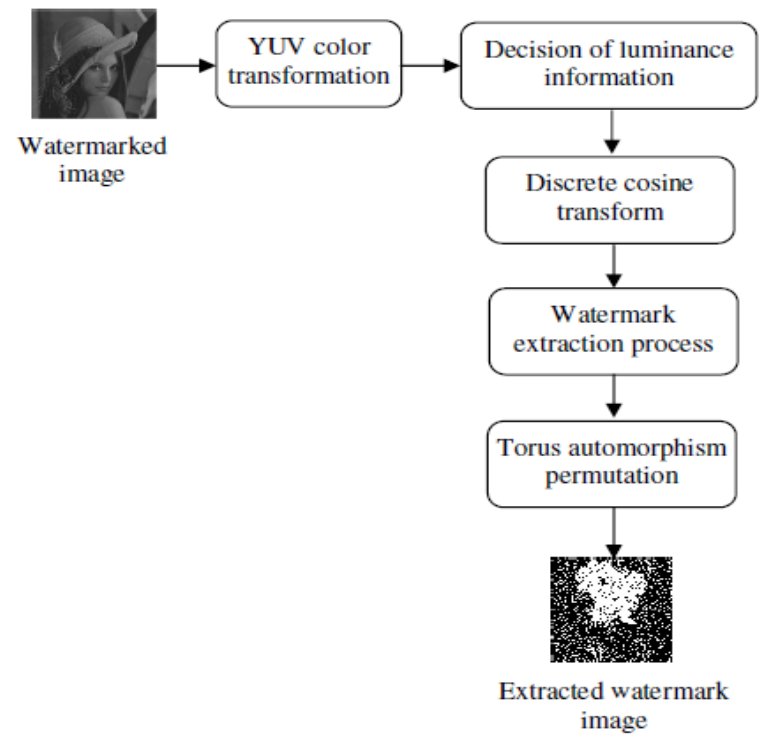

Fig. 4: Watermark Extraction process

The block diagram of the watermark extraction process is given in Fig. 4.

\section{MATERIALS AND METHODS}

This section describes the experimental results and comparative analysis of four considered algorithms. These algorithms are implemented using MATLAB. Here, for comparison, we have taken the watermark image and the host images such as, Lena, Baboon and Cameraman. For each input host images, the watermark image is embedded based on embedding algorithms and the watermark image is extracted from the watermarked image using extraction algorithms. The outputs obtained from the different watermarking algorithms are compared based on the metrics, for example, PSNR and NC. The visual quality is evaluated for the algorithms by the Peak Signal-To-Noise Ratio (PSNR) criterion in between the host images with the watermarked images and the extracting fidelity is compared by the Normalized Correlation (NC) value between the original watermark and the extracted watermark. The definition of PSNR and NC is given in the following equations:

PSNR $=10 \log _{10} \frac{E_{\max }^{2} \times I_{w} \times I_{h}}{\sum\left(I_{x y}-I_{x y}^{*}\right)}$

Where:

$\mathrm{I}_{\mathrm{w}}$ and $\mathrm{I}_{\mathrm{h}}=$ Width and height of the watermarked image 
Am. J. Applied Sci., 8 (1): 63-70, 2011

$$
\begin{aligned}
\mathrm{I}_{\mathrm{xy}} & =\text { Original image pixel value at coordinate } \\
& (\mathrm{x}, \mathrm{y}) \\
\mathrm{I}_{\mathrm{xy}}^{*} & =
\end{aligned}
$$$$
N C=\frac{\sum_{i=0}^{N} \sum_{j=0}^{M} W(i, j) \times W *(i, j)}{\sum_{i=0}^{N} \sum_{j=0}^{M}[W(i, j)]^{2}}
$$

Where:

$\mathrm{W}(\mathrm{i}, \mathrm{j}) \quad=$ Original watermark image

$\mathrm{W}^{*}(\mathrm{i}, \mathrm{j})=$ Extracted watermark image

$\mathrm{N}$ and $\mathrm{M}=$ Width and height of the watermark image

\section{RESULTS}

The robustness of the considered algorithms can be evaluated with the help of compression techniques for JPEG. The algorithm is said to be robust only if the considered algorithms can be able to extract the watermark information after applying the compression in the watermarked image. Here, we apply the different compression standard in the watermarked images to analyze the robustness of the watermarking algorithms in terms of visual quality and the fidelity.

JPEG: JPEG is a lossy compression algorithm that has been constructed for the purpose of decreasing the file size of natural, photographic-like true-color images to the maximum possible extent without degrading the visual quality of the image in terms of human perception. For robustness comparison, we make use of JPEG compression that is applied in the watermarked images, the watermark image is extracted and PSNR and NC metrics are calculated for various compression ratios to evaluate the performance of the watermarking algorithms.

Peak signal-to-noise ratio: The PSNR of the considered algorithms are computed for various compression ratios of JPEG compression. The values taken for different algorithms are plotted as a graph shown in Fig. 5-7. Here, the watermarking algorithm proposed by Ye and Tan has achieved highest PSNR for compression ratio for example, 90 and 100 rather than the baboon image. But, Liu et al. (2006) algorithm outperformed all other algorithms for the highly compressed images. The PSNR obtained by the Lin et $a l$. algorithm is behaved similarly for the entire input images as well as the output is independent of the compression ratio.

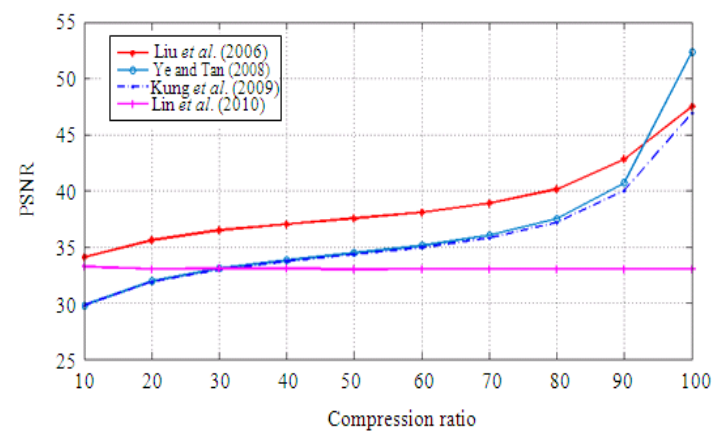

Fig. 5: PSNR obtained by the different algorithms for Lena image

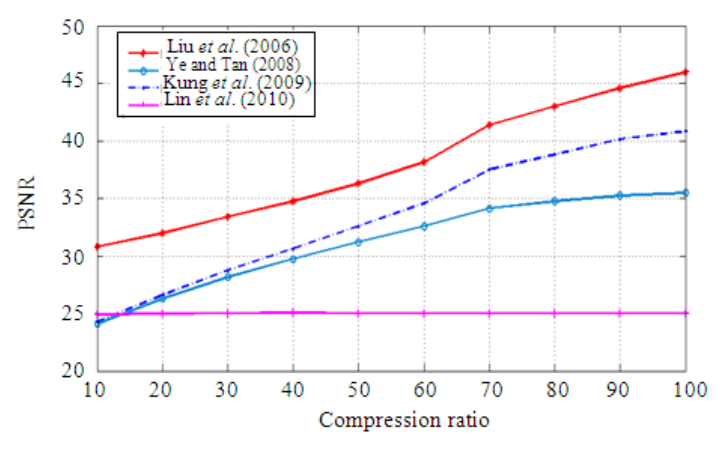

Fig. 6: PSNR obtained by the different algorithms for Baboon image

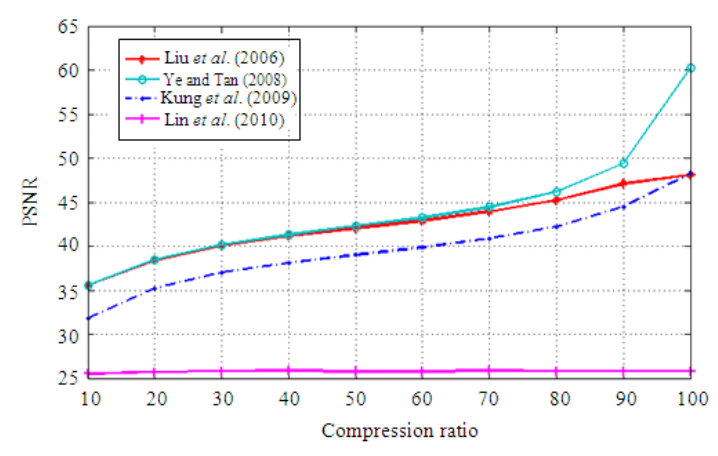

Fig. 7: PSNR obtained by the different algorithms for Cameraman image

Normalized correlation: The comparison of considered algorithms based on Normalized Correlation is given in this sub-section. The NC values are computed for different compression ratios of JPEG compression and the values are plotted as a graph shown in Fig. 8-10 for the considered algorithms. The behavior of the algorithm proposed by Ye and Tan is changed according to the input host images. Again, Liu et al. algorithm achieved almost highest NC for all host images except baboon image. 
Am. J. Applied Sci., 8 (1): 63-70, 2011

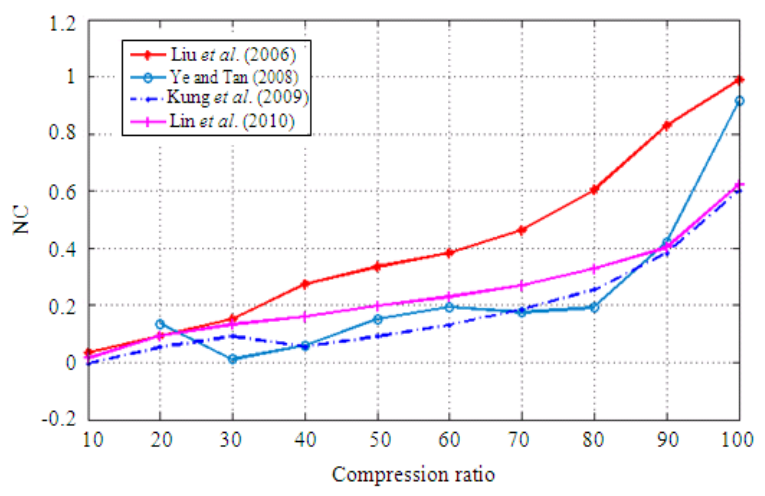

Fig. 8: NC obtained by the different algorithms for Lena image

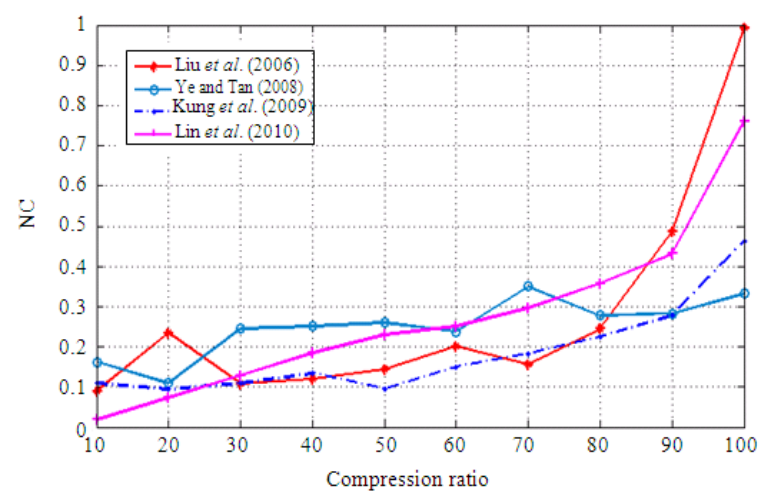

Fig. 9: NC obtained by the different algorithms for Baboon image

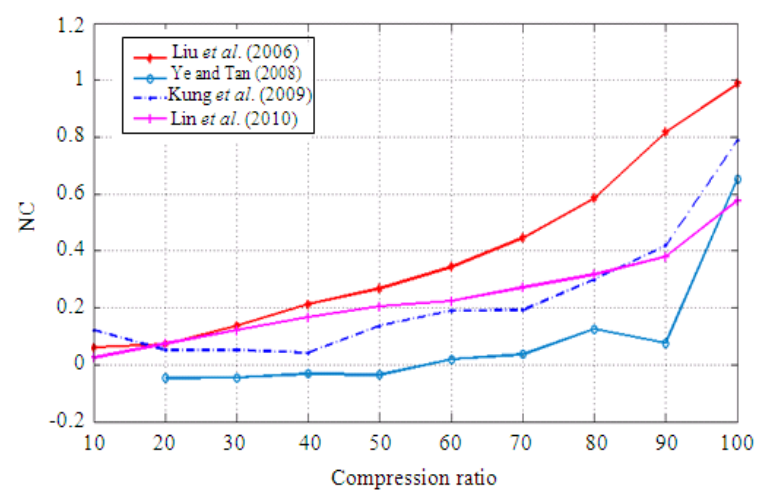

Fig. 10: NC obtained by the different algorithms for Cameraman image

\section{DISCUSSION}

In this study, we presented an extensive analysis of four robust watermarking algorithms done in the frequency domain. The performance study was carried out on four watermarking algorithms in terms of visual quality and the fidelity. Furthermore, the behavior of the watermarking algorithm was analyzed after applying the various compressions in the watermarked images. The comparative study has revealed that Liu et al. algorithm achieved better visual quality when compared with other algorithms. Moreover, the experimental results have shown that Liu et al. algorithm was better (in terms of fidelity) of the other algorithms. The algorithm proposed by Lin et al. has not significantly changed the visual quality according to the compression ratio.

\section{CONCLUSION}

As demonstrated in the experimental results, the proposed intellectual property rights protection scheme can visually identify the owner of image, the signature can be completely retrieved from the compressed watermarked image and the falsification position within the image can be detected when the image is falsified. Therefore, we conclude that the new proposed technique is more suitable for images that will be highly JPEGcompressed and transmitted on the Internet.

\section{REFERENCES}

Bankovic, Z., J.M. Moya, A. Araujo, S. Bojanic and O. Nieto-Taladriz, 2009. A genetic algorithmbased solution for intrusion detection. J. Inform. Assurance Secur., 4: 192-199.

Bohra, A., O. Farooq and Izharuddin, 2009. Blind selfauthentication of images for robust watermarking using integer wavelet transform. AEU Int. J. Elect. Commun., 63: 703-707. DOI: 10.1016/J.AEUE.2008.05.010

Chandra, N.S., M.B. Shaik, A. Govardhan and M. Balraju, 2010. Comparative study of the effect of lossy and lossless block based binary plane techniques on medical images. J. Comput. Sci., 6: 1377-1380. DOI: 10.3844/jcssp.2010.1377.1380

Coria, L., P. Nasiopoulos, R. Ward and M. Pickering, 2007. An access control video watermarking method that is robust to geometric distortions. J. Inform. Assurance Secur., 2: 266-274.

Cox, I.J., M.L. Miller and J.A. Bloom, 2002. Digital Watermarking. 1st Edn., Morgan Kaufmann, USA., ISBN-10: 1558607145, pp: 542.

Kumar, S., B. Raman and M. Thakur, 2009. Real coded genetic algorithm based stereo image watermarking. IJSDIA Int. J. Secure Digital Inform. Age, 1: 23-33. 
Kung, C.M., S.T. Chao, Y.C. Tu, Y.H. Yan and C.H. Kung, 2009. A robust watermarking and image authentication scheme used for digital contant application. J. Multimedia, 4: 112-119. DOI: 10.4304/jmm.4.3.112-119

Lai, C.-C., H.-C. Huang and C.-C. Tsai, 2009. A digital watermarking scheme based on singular value decomposition and micro-genetic algorithm. Int. J. Innov. Comput., Inform. Control, 5: 1867-1873.

Langelaar, G.C., I. Setywan and R.L. Lagendijk, 2000. Watermarking digital image and video data. A state-of-the-art overview. IEEE Signal Process. Mag., 17: 20-46. DOI: 10.1109/79.879337

Lin, S.D., S.C. Shie and J.Y. Guo, 2010. Improving the robustness of DCT-based image watermarking against JPEG compression. Comput. Stand. Interfaces, $\quad 32: \quad 54-60 . \quad$ DOI: 10.1016/J.CSI.2009.06.004

Liu, J.L., D.C. Lou, M.C. Chang and H.K. Tso, 2006. A robust watermarking scheme using self-reference image. Comput. Stand. Interfaces, 28: 356-367. DOI: 10.1016/J.CSI.2005.07.001

Lu, W., H. Lu and F.-L. Chung, 2010. Feature based robust watermarking using image normalization. Comput. Elect. Eng., 36: 2-18. DOI: 10.1016/J.COMPELECENG.2009.04.002

Marimuthu, C.N. and P. Thangaraj, 2010. Low power multiplier design using latches and flip-flops. J. Comput. Sci., 6: 1117-1122. DOI: 10.3844/jcssp.2010.1117.1122

MeenakshiDevi, P., M. Venkatesan and K. Duraiswamy, 2009. A fragile watermarking scheme for image authentication with tamper localization using integer wavelet transform. J. Comput. Sci., 5: 831-837.
Peng, H., J. Wang and W. Wang, 2009. Adaptive image watermarking approach based on kernel clustering and HVS. Proceedings of the 8th International Workshop on Fuzzy Logic and Applications, (IWFLA’09), Springer-Verlag Berlin, Heidelberg, New York, pp: 213-220.

Petitcolas, F.A.P., R.J. Anderson and M.G. Kuhn, 1999. Information hiding-a survey. Proceed. IEEE, 87: 1062-1078. DOI: 10.1109/5.771065

Piotrowski, Z. and P. Gajewski, 2007. Voice spoofing as an impersonation attack and the way of protection. J. Inform. Assurance Secur., 2: 223-225.

Sarhan, A.M., 2009. Iris recognition using discrete cosine transform and artificial neural networks. J. Comput. Sci., 5: 369-373. DOI: 10.3844/jcssp.2009.369.373

Surekha, B., G.N. Swamy, K.S. Rao and A.R. Kumar, 2009. A watermarking technique based on visual cryptography. J. Inform. Assurance Secur., 4: 470-473.

Szczypiorski, K., I. Margasinski and W. Mazurczyk, 2007. Steganographic routing in multi agent system environment. J. Inform. Assurance Secur., 2: 235-243.

Wang, X.-Y. and H. Zhao, 2006. A novel synchronization invariant audio watermarking scheme based on DWT and DCT. IEEE Trans. Signal Process., 54: 4835-4840. DOI: 10.1109/TSP.2006.881258

Ye, J.X. and G.Z. Tan, 2008. An improved digital watermarking algorithm for meaningful image. Proceedings of International Conference on Computer Science and Software Engineering, Dec. 12-14, Wuhan, Hubei, pp: 822-825. 